\title{
LOCAL CONNECTEDNESS AND PSEUDOCONIPACTNESS IN COMPLETELY REGULAR SPACES
}

\author{
DONALD G. HARTIG
}

\begin{abstract}
The properties of local connectedness and pseudocompactness of a completely regular space $X$ are characterized via algebraic properties of the space $C(X)$. These characterizations are then used to prove the (wellknown) theorem that $\beta X$ is locally connected if and only if $X$ is locally connected and pseudocompact.
\end{abstract}

1. $X$ is a completely regular Hausdorff space, $C(X)$ is the space of all bounded, continuous, real-valued functions on $X$ and $\beta X$ is the Stone-Čech compactification of $X$. For $f \in C(X),\|f\|$ denotes its supremum norm and $\operatorname{coz} f$ is its cozero set, i.e. $\operatorname{coz} f=\{x \in X: f(x) \neq 0\}$. It is the purpose of this note to give characterizations of pseudocompactness and of local connectedness of $X$ in terms of properties of $C(X)$ as an algebra of functions on $X$. These characterizations will then be used to prove the following theorem (due to Banaschewski [1] and Henriksen and Isbell [4]).

THEOREM. $\beta X$ is locally connected if and only if $X$ is locally connected and pseudocompact.

Characterizations similar to those offered here have been given. The paper [3] by Glicksburg contains several interesting characterizations of pseudocompactness as do the monographs [2] and [5]. In what follows we will use the following characterization found in [5], see also [6, Theorem 10.2.5].

LEMMA. $X$ is pseudocompact if and only if every neighborhood-finite family of open sets in $X$ is finite.

D. Wulbert [7] has given a proof of the above theorem via a characterization of local connectedness of $X$ in terms of the order and norm structure of $C(X)$. An exposition appears in [5]. Our characterization uses only the algebraic structure of $C(X)$ but the proof of the theorem relies on the norm structure as well.

$\$ 2$ contains some definitions and the characterization of pseudocompactness. The characterization of local connectedness and the proof of the Banaschewski-Henriksen-Isbell Theorem is given in \$3.

Presented to the Society, January 29, 1977; received by the editors February 14, 1977 and, in revised form, June 23, 1977.

AMS (MOS) subject classifications (1970). Primary 54C40, 54D05, 54D35; Secondary 46E25.

Key words and phrases. Completely regular, locally connected, pseudocompact, $C(X)$, pointwise convergent, uniform convergent, Stone-Cech compactification.

(c) American Mathematical Society 1978 
2. Two functions $f$ and $g$ in $C(X)$ will be called disjoint when coz $f \cap \operatorname{coz} g$ $=\varnothing$. This is equivalent to asking that $f g=0$ so that $f$ and $g$ are disjoint in $C(X)$ if and only if their extensions $\bar{f}$ and $\bar{g}$ are disjoint in $C(\beta X)$. A function $f \in C(X)$ will be called indecomposable when it cannot be written (nontrivially) as the sum of two disjoint functions in $C(X)$. Thus $f$ is indecomposable in $C(X)$ if and only if $\bar{f}$ is indecomposable in $C(\beta X)$. An easy argument will show that $f$ is indecomposable if and only if $\operatorname{coz} f$ is connected.

A family $\mathscr{F}$ of functions in $C(X)$ will be called disjoint when any two functions in $\mathscr{F}$ are disjoint. Notice that $\mathscr{F}$ is disjoint in $C(X)$ if and only if $\overline{\mathscr{F}}=\{\bar{f}: f \in \mathscr{F}\}$ is disjoint in $C(\beta X)$. Now given $\mathscr{F}$ disjoint in $C(X)$ we can define a new function $\bigoplus \mathscr{F}$ on $X$ in a natural way. Its value at $x \in X$ is $\Sigma\{f(x): f \in \mathcal{F}\}$, this sum being only apparently infinite. The function $\bigoplus \mathcal{F}$ is, in general, neither continuous nor bounded on $X$. However if $\{\operatorname{coz} f$ : $f \in \mathscr{F}\}$ is neighborhood-finite then $\bigoplus \mathscr{F}$ is continuous.

$\bigoplus \mathscr{F}$ is the point-wise sum of the family $\mathcal{F}$ and should not be mistaken for the uniform sum which, when it exists, will be denoted $\Sigma \mathcal{F}$. Recall that $f=\Sigma \mathscr{F}$ when, given any $\varepsilon>0$, there exists a finite subfamily $F$ of $\mathscr{F}$ such that $\|f-\bigoplus G\|<\varepsilon$ whenever $F \subset G \subset \mathscr{F}, G$ finite. If $\Sigma \mathscr{F}$ exists it is both continuous and bounded.

It is clear that if $f \in C(X)$ and $f=\sum \mathscr{F}$ for some disjoint family $\mathscr{F}$, then $f=\bigoplus \mathscr{F}$. The converse is generally false as the following theorem shows.

THEOREM 1. The following two conditions are equivalent:

(1) Given $f \in C(X)$ and a disjoint family $\mathscr{F}$ in $C(X), f=\bigoplus \mathscr{F}$ implies $f=\sum \mathscr{F}$.

(2) $X$ is pseudocompact.

Proof. (1) implies (2). If $X$ is not pseudocompact a simple argument will produce a neighborhood-finite family of nonempty, disjoint, open sets $\left\{U_{n}\right\}_{n=1}^{\infty}$ (see [6]). For each $n$ we obtain a function $f_{n} \in C(X)$ such that $0<f_{n} \leqslant 1,\left\|f_{n}\right\|=1$ and $\operatorname{coz} f_{n} \subset U_{n}$. Letting $\mathscr{F}=\left\{f_{n}\right\}_{n=1}^{\infty}$ the function $f=\bigoplus \mathscr{F}$ is in $C(X)$ but $f \neq \sum \mathscr{F}$.

(2) implies (1). Assume $X$ is pseudocompact. Let $f=\bigoplus \mathscr{F}$ where $f \in C(X)$ and $\mathscr{F}$ is disjoint in $C(X)$. Given $\varepsilon>0$ let $F$ denote the family of functions $g \in \mathscr{F}$ such that

$$
\{x \in X:|g(x)|>\varepsilon\} \neq \varnothing \ldots
$$

The family of all open sets described by $(*)$ is neighborhood-finite; hence finite by pseudocompactness. Thus $F$ is finite and clearly $\|f-\bigoplus G\|<\varepsilon$ whenever $F \subset G \subset \mathscr{F}, G$ finite.

The above might be called a Dini-type characterization of pseudocompactness. Indeed Glicksburg showed that $X$ is pseudocompact if and only if Dini's Theorem holds in $C(X)$. 
3. The reader can supply the argument showing that, given $f \in C(X)$, the connected components of $\operatorname{coz} f$ are open if and only if $f=\bigoplus \mathscr{F}$ for a disjoint family $\mathscr{F}$ of indecomposable functions in $C(X)$. This observation together with the fact that the cozero sets in $X$ form a basis for the topology constitutes the proof of the following theorem.

THEOREM 2. $X$ is locally connected if and only if given any $f \in C(X)$ there is a disjoint family $\mathscr{F}$ of indecomposable functions in $C(X)$ such that $f=\bigoplus \mathscr{F}$.

We will now use Theorems 1 and 2 to give an elementary proof of the theorem stated in $\$ 1$.

Proof of Banaschewski-Henriksen-Isbell Theorem. Assume first that $X$ is locally connected and pseudocompact. Given $f \in C(X)$ Theorems 1 and 2 combine to imply that $f=\Sigma \mathscr{F}$ for a disjoint family $\mathscr{F}$ of indecomposable functions in $C(X)$. Extending to $C(\beta X)$ we have $\bar{f}=\Sigma \overline{\mathscr{F}}$ and $\overline{\mathscr{F}}$ is also disjoint with indecomposable members. Since $\bar{f}$ is a typical member of $C(\beta X)$ Theorem 2 implies $\beta X$ is locally connected.

Now assume $\beta X$ is locally connected. $X$ can be shown to be locally connected with an argument similar to the one above. We show $X$ is pseudocompact via Theorem 1. Let $f=\bigoplus \mathscr{F}$ where $f \in C(X)$ and $\mathscr{F}$ is a disjoint family in $C(X)$. Since $\beta X$ is locally connected and compact there is a disjoint family $\mathcal{G}$ of indecomposable functions in $C(X)$ such that $\bar{f}=\Sigma \overline{\mathcal{G}}$. It follows that $f=\Sigma \mathcal{G}$. Now, because, for each $g \in \mathcal{G}$, $\operatorname{coz} g$ is connected there is a unique $g^{\prime} \in \mathcal{F}$ such that $\operatorname{coz} g \subset \operatorname{coz} g^{\prime}$. Let $G \subset \mathcal{G}$ be finite with $\|f-\bigoplus G\|<\varepsilon$, then $G^{\prime}=\left\{g^{\prime}: g \in G\right\}$ is a finite subset of $\mathscr{F}$ having the same property and since $\|f-\bigoplus F\| \leqslant\left\|f-\bigoplus G^{\prime}\right\|$ for any finite $F$ with $G^{\prime} \subset F \subset \mathscr{F}$ we have $f=\Sigma \mathscr{F}$.

REMARK. It is tempting to shorten the above proof by replacing the last three sentences with: "We have $\bar{f}=\bigoplus \overline{\mathscr{F}}$ and since $\beta X$ is compact, $\bar{f}=\Sigma \overline{\mathscr{F}}$ implying $f=\Sigma \mathscr{F}$." However, since no use of local connectedness is made, this argument is spurious. We leave it to the reader to decide where it fails.

Theorems 1 and 2 will also combine to give the following normed algebra characterization of local connectedness in a compact space.

THEOREM 3. The compact Hausdorff space $X$ is locally connected if and only if given any $f \in C(X)$ there is a disjoint family $\mathscr{F}$ of indecomposable functions in $C(X)$ such that $f=\Sigma \mathscr{F}$.

\section{REFERENCES}

1. B. Banaschewski, Local connectedness of extension spaces, Canad. J. Math. 8 (1956), 385-388.

2. L. Gillman and M. Jerison, Rings of continuous functions, University Series in Higher Math., Van Nostrand, Princeton, N. J., 1960.

3. J. Glicksburg, The representation of functionals by integrals, Duke Math. J. 19 (1952), 253-261.

4. M. Henriksen and J. Isbell, Local connectedness in the Stone-Cech compactification, Illinois J. Math. 1 (1957), 574-582. 
5. R. Walker, The Stone-Čech compactification, Ergebnisse der Mathematik, Grenzgebiete und ihrer Grenzgebiete, Band 83, Springer-Verlag, Berlin and New York, 1974.

6. A. Wilansky, Topology for analysis, Ginn, Waltham, Mass., 1970.

7. D. Wulbert, $A$ characterization of $C(X)$ for locally connected $X$, Proc. Amer. Math. Soc. 21 (1969), 269-272.

Department of Mathematics, Untred States Naval Academy, Annapolis, Maryland 21402 